\title{
Analysis of clinical data of viral encephalitis patients complicated with epilepsy during the acute phase
}

\author{
Shi-Ying Chen ${ }^{1}$, Wei Ma², Yan-Yan Chen 1 , Xue-Wen Fan² \\ ${ }^{1}$ Department of Neurology, Ningxia Medical University, Yinchuan 750004, Ningxia, China. \\ ${ }^{2}$ Department of Neurology, the Cardia-Cerebrovascular Disease Hospital of Ningxia Medical University, Yinchuan 750004, Ningxia, China.
}

Correspondence to: Prof. Xue-Wen Fan, Department of Neurology, the Cardia-Cerebrovascular Disease Hospital of Ningxia Medical University, Yinchuan 750004, Ningxia, China. E-mail: nxfxw@163.com

How to cite this article: Chen SY, Ma W, Chen YY, Fan XW. Analysis of clinical data of viral encephalitis patients complicated with epilepsy during the acute phase. Neuroimmunol Neuroinflammation 2017;4:124-31.

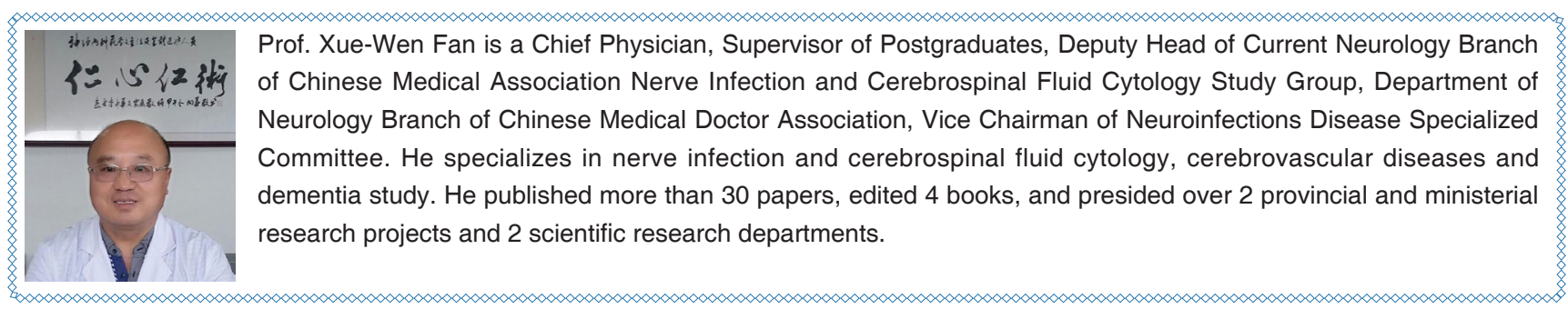

Article history:

Received: 29-03-2017

Accepted: 10-05-2017

Published: 11-07-2017

Key words:

Viral encephalitis,

epilepsy,

treatment,

clinical characteristics

\section{ABSTRAC T}

Aim: To compare the difference between viral encephalitis patients complicated with epilepsy group and those without epilepsy. Methods: The authors retrospectively study 116 cases of viral encephalitis patients admitted to the General Hospital of Ningxia Medical University and the Cardia-Cerebrovascular Disease Hospital of Ningxia Medical University from January 2011 to December 2016. There were 39 cases with epilepsy and 77 cases without epilepsy. By surveying the Hospital Information System, the authors collected their clinical data including general situations, medical history, physical examination, clinical presentation, laboratory examination, cerebrospinal fluid examination, imaging examination, electroencephalogram examination (EEG), treatment, and discharge conditions. The authors used SPSS for further analysis and statistics. Results: Medical history: there were significant differences between the two groups in disturbance of consciousness, cognitive dysfunction, and admission conditions $(P<0.05)$. No significant differences were found in other clinical manifestations. Auxiliary examination: in cerebrospinal fluid (CSF) examination, the patients with epilepsy had higher glucose $(P<0.05)$. Brain imaging examination shows that cortical involvements in patients with epilepsy were higher $(P<0.05)$, and EEG examination showed that patients with epilepsy were more severe $(P<0.05)$. No significant differences were found in other auxiliary 
examination. Treatment and prognosis: all the patients were given antiviral treatment. Twenty-eight cases of the patients with epilepsy (71.79\%) are used of antiepileptic drugs. The prognosis of patients with epilepsy was poor $(P<0.05)$. Conclusion: Viral encephalitis frequently manifests with seizures in its acute phase, and the incidence of seizures in our study was $33.62 \%$. The main form was generalized seizures $(64.10 \%)$, which could occur at any age; patients with epilepsy had a higher cortical involvement on imaging and a higher degree of EEG abnormalities than patients without; patients with epilepsy had a higher level of glucose in the CSF; an episode of viral encephalitis complicated with seizures could aggravate disturbances of consciousness and cognitive impairment. The prognosis of patients with epilepsy was poor.

\section{INTRODUCTION}

Viral encephalitis refers to an acute inflammatory process of the brain parenchyma due to direct viral infection. The estimated incidence of clinicallydiagnosed viral encephalitis is 3.5-7.4/100,000/year. ${ }^{[1]}$ At present, we have found more than 130 viruses that can cause viral encephalitis. The common viruses found in the clinic are the herpes simplex virus Type-I, Type-II (HSV-I, II), varicella-zoster virus, Japanese encephalitis virus, and enterovirus (mainly for ECHO virus and Coxsackie virus). Among the sporadic viral encephalitides, herpes simplex encephalitis (HSE) is perhaps the most frequently associated with epilepsy, which may often be severe. Among the epidemic (usually due to flaviviruses) viral encephalitides, Japanese encephalitis (JE) is the most common and is associated with acute symptomatic seizures, especially in children. ${ }^{[2]}$

There are many methods for detection of viral encephalitis, but they all have advantages and disadvantages. Even with the best efforts, 30-60\% of patients with clinically suspected viral encephalitis still cannot be diagnosed. Virological diagnosis is the "gold standard." But isolation of the virus is seldom accomplished due to the difficulty in obtaining brain tissue through biopsy and a lack of specialized facilities for viral cultures in many places. Moreover, there were greater complications of brain biopsy. Virus antibody testing in serum or cerebrospinal fluid (CSF) are simpler to perform and more widely available than brain biopsy, but it has low sensitivity because many viruses do not express specific antibodies. Virus nucleic acid testing has higher sensitivity and specificity. Polymerase chain reaction (PCR) technology has significantly improved virological diagnosis. But many laboratories fail to meet the technical requirements and, thus, restrict the widespread application in this area of clinical microbiology. Therefore, the diagnosis of viral encephalitis currently depends on cerebrospinal fluid examination, brain magnetic resonance imaging (MRI) or computed tomography (CT), and electroencephalogram (EEG). Cerebrospinal fluid examination manifests with normal or elevated encephalic pressure, normal or mildly elevated protein, and normal sugar and chloride in most cases. The numeration of leukocyte is $(50-500) \times$ $10^{6} / \mathrm{L}$ in the cytological examination and most of them are lymphocytes. Cerebrospinal fluid examination has important significance for distinguishing other types of encephalitis..$^{[3]}$ The appearances on early EEG are mainly abnormal rhythm and increased slow wave in viral encephalitis patients. ${ }^{[4]}$ CT shows foci mainly located in one or both temporal lobes and single or multiple low density foci. CT is currently used only for infant patients and patients who do not cooperate or who move restlessly. MRI is the most important imaging examination. The edge of an abnormal area on a routine MRI scan is often unclear and may include lesions and peripheral edema. It will show a long T1 and long T2 signal and a short T1 and long T2 when it is accompanied by hemorrhage. Lesions also show high signal on diffusion weighted imaging (DWI). An enhanced MRI may have a higher sensitivity for immune compromised patients and mild patients and may include blood brain barrier damage [Figures 1 and 2]. ${ }^{[5]}$

Viral encephalitis frequently manifests with seizures in its acute phase with an incidence of $19.0-62.69 \%$. ${ }^{[6,7]}$ Epileptic seizures may aggravate brain parenchyma damage and induce many severe complications that affect prognosis and quality of life, such as: pulmonary infectious disease; respiration-circulation failure; fractures and tongue biting in patients with general tonic-clonic seizures; and water-electrolyte imbalance in patients with status epilepticus. So far the researches of viral encephalitis complicated with epilepsy are not completely consistent. Herein, we retrospectively studied 116 cases of viral encephalitis and compared the difference between those complicated with epilepsy and those without. As a result, we are able to diagnosis early and take effective treatment to reduce mortality and improve prognosis.

\section{METHODS}

\section{Subject investigated}

We retrospectively studied 116 cases of viral encephalitis admitted to the General Hospital of Ningxia Medical University and the Cardio-Cerebrovascular Disease Hospital of Ningxia Medical University from January 2011 to December 2016. The inclusion 
criteria: ${ }^{[8]}$ (1) acute or subacute onset, some patients had a history of prodromal infections; (2) patients had symptoms of brain parenchymal damage; (3) cerebrospinal fluid examinations were consistent with viral encephalitis; (4) there was brain parenchymal damage or brain edema on imaging and varying degrees of alteration in EEG. The exclusion criteria: (1) patients younger than 14 years were excluded; (2) patients with history of epilepsy or other secondary epilepsy were excluded; (3) we removed other explicit epileptogenic causes such as hypoxia, blood glucose abnormalities, poisoning, uremic damage and so on; (4) the main clinical materials were incomplete.

\section{Content}

We collected, through a survey of the Hospital Information System, the clinical data of 116 cases of viral encephalitis including general situation, medical history, physical examination, clinical presentation, laboratory examination, cerebrospinal fluid examination, imaging examination, EEG examination, treatment, and discharge conditions

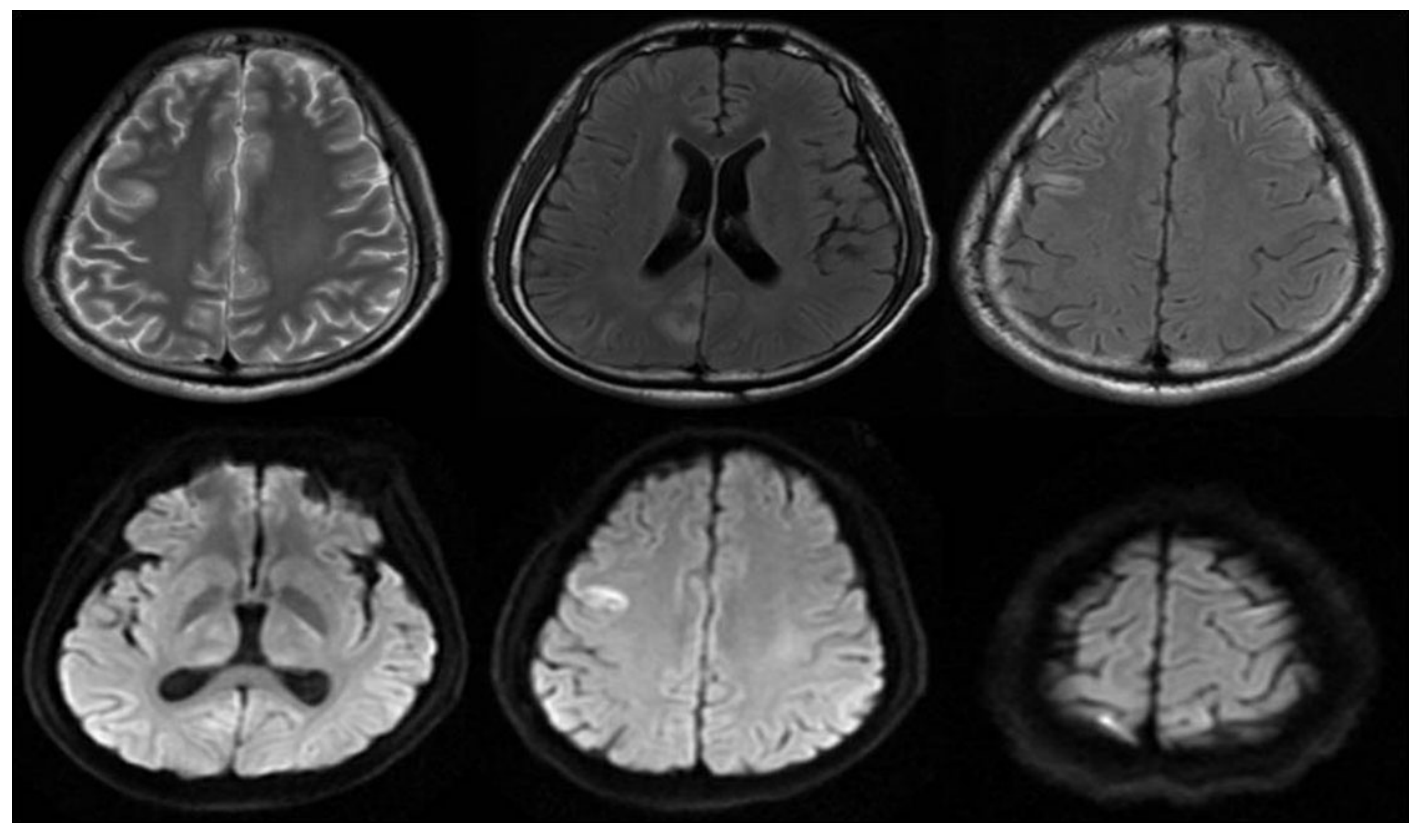

Figure 1: Abnormal signal can be seen in the right frontal lobe, occipital lobe and bilateral dorsal thalamus, caudate nucleus in the brain magnetic resonance imaging, shows high signal on T2WI and a slightly higher signal on Flair, shows high signal diffusion weighted imaging

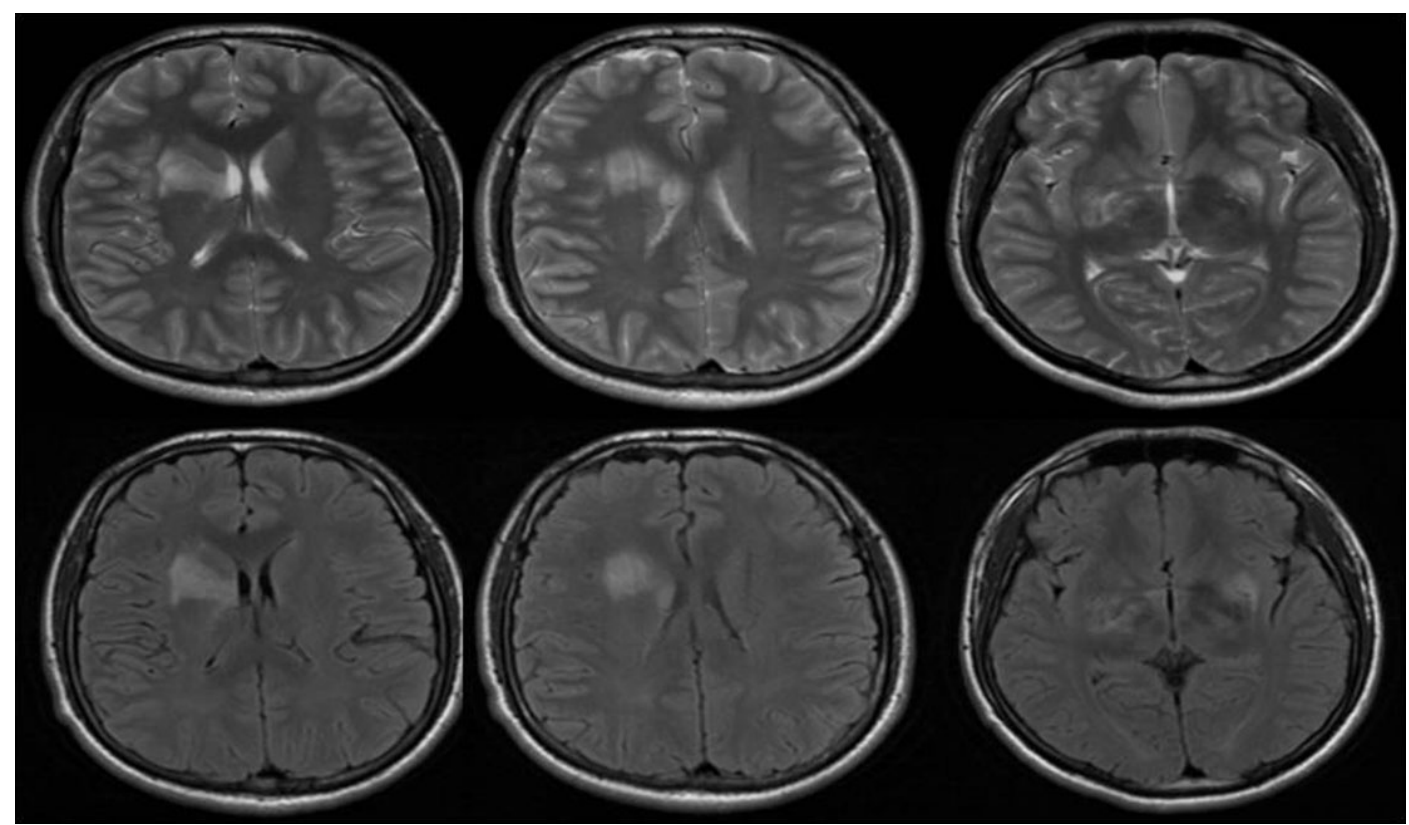

Figure 2: Abnormal signal can be seen in bilateral hippocampus and right putamen in the brain magnetic resonance imaging, shows high signal on T2WI and high signal on Flair 
and analyzed the data.

\section{Statistical methods}

We used SPSS 17.0 for statistical and further analysis. The measurement data and enumeration data were statistically analyzed with Rank Sum test and $\chi^{2}$ test respectively.

\section{RESULTS}

\section{General situations}

In the prior 6 years, the incidence of viral encephalitis had not increased obviously. There were 67 cases of male patients $(57.76 \%)$ and 49 cases of female patients $(42.24 \%)$ among all the patients. The mean age of presentation was $41.00 \pm 19.06$ years in the group with epilepsy and $37.70 \pm 15.59$ years in the group without epilepsy. The average length of stay was $19.53 \pm 16.46$ days in the group with epilepsy and $19.84 \pm 12.76$ days in the group without epilepsy. There were no significant differences between the two groups in gender, nationality, age, average hospitalization days; however, there were significantly differences in the conditions upon entering the hospital $(P=0.020)$ [Table 1].

\section{Clinical presentation}

Among the 116 patients with viral encephalitis, 46 cases had definite causes. Catching cold was the main precipitating factor for 41 cases $(89.13 \%)$. Two cases $(4.34 \%)$ were precipitated by psychiatric and psychological factors; 2 cases $(4.34 \%)$ were precipitated by overwork; and 1 case was precipitated by brain trauma. There were 96 patients $(82.76 \%)$ with acute onset and 20 patients (17.24\%) with subacute onset. Some viral encephalitides have a history of prodromal infections, such as, respiratory infections (especially upper respiratory tract infections), digestive tract infections, and mucocutaneous herpes. Part of the patients were complicated with severe pneumonia, atrial fibrillation, heart failure and other cardiovascular diseases, gastrointestinal bleeding, and other serious medical diseases [Table 2].

There were 7 cases of patients $(6.03 \%)$ with initial symptoms of epilepsy, which is much lower than that of Misra's study of $30.7 \%$ vs. $42.6 \%{ }^{[7]}$ Possible reasons are that patients and their families do not recognize partial epilepsy seizure, there are no family members in the presence of seizures, and the patients cannot provide the relevant medical history themselves. There were 3 cases of patients $(2.59 \%)$ with initial symptom of dizziness, nausea, vomiting and other general symptoms; 1 case of patients $(0.86 \%)$ with initial symptom of visual disorder; 2 cases of patients $(1.72 \%)$ with initial symptom of hemiplegia; 2 cases of patients $(1.72 \%)$ with initial symptom of paresthesia; 1 case of patients $(0.86 \%)$ with initial symptom of ataxia; and 1 case of patients $(0.86 \%)$ with initial symptom of conscious disturbance. The main manifestations of viral encephalitis include headache, fever, seizures, limb weakness, consciousness disturbance, mental and behavioral disorders, language disorders, cognitive dysfunction, ataxia, and varying degrees of alteration

Table 1: Comparison of gender, nationality and entering hospital condition between the two groups

\begin{tabular}{|c|c|c|c|c|c|c|}
\hline \multirow{2}{*}{ Characteristics } & \multicolumn{2}{|c|}{ With epilepsy group } & \multicolumn{2}{|c|}{ Without epilepsy group } & \multirow{2}{*}{$\chi^{2}$} & \multirow{2}{*}{$P$} \\
\hline & Case & Rate (\%) & Case & Rate (\%) & & \\
\hline Male & 23 & 58.97 & 44 & 57.14 & 0.036 & 0.850 \\
\hline Female & 16 & 41.02 & 33 & 42.85 & & \\
\hline Han nationality & 28 & 71.79 & 57 & 74.02 & 0.066 & 0.798 \\
\hline Non-Han nationality & 11 & 28.20 & 20 & 25.97 & & \\
\hline Common patients & 10 & 25.64 & 37 & 48.05 & 5.395 & 0.020 \\
\hline Severe patients & 29 & 74.35 & 40 & 51.95 & & \\
\hline
\end{tabular}

Table 2: Comparison of clinical manifestations between the two groups

\begin{tabular}{lcccccc}
\hline \multirow{2}{*}{ Clinical manifestations } & \multicolumn{2}{c}{ With epilepsy group } & \multicolumn{2}{c}{ Without epilepsy group } & \multirow{2}{*}{$\chi^{2}$} & \multirow{2}{*}{$\boldsymbol{P}$} \\
\cline { 2 - 5 } & Case & Rate (\%) & Case & Rate (\%) & & \\
\hline Headache & 30 & 76.92 & 66 & 85.71 & 1.402 & 0.236 \\
Fever & 31 & 79.49 & 55 & 71.43 & 0.877 & 0.349 \\
Consciousness disturbance & 32 & 82.05 & 16 & 20.78 & 40.067 & 0.001 \\
Cognitive dysfunction & 24 & 61.54 & 28 & 36.26 & 6.634 & 0.01 \\
Sensory disturbance & 2 & 5.13 & 4 & 5.19 & 0.000 & $1.000^{*}$ \\
Involuntary movement & 3 & 7.69 & 2 & 2.60 & 0.628 & $0.428^{*}$ \\
Decreased memory & 11 & 28.21 & 12 & 15.58 & 2.594 & 0.107 \\
Language disorders & 11 & 28.21 & 12 & 15.58 & 2.594 & 0.107 \\
Autonomic nervous dysfunction & 4 & 10.26 & 4 & 5.19 & 0.395 & $0.530^{*}$ \\
Central hypoventilation & 8 & 20.51 & 6 & 7.79 & 2.84 & $0.092^{*}$ \\
Meningeal irritation sign & 12 & 30.77 & 19 & 24.68 & 0.491 & 0.484 \\
\hline
\end{tabular}

*stands for using the chi square test for correction 
in sensorium. There were significant indications between the two groups in consciousness disturbance $(P=0.001)$ and cognitive dysfunction $(P=0.01)$, both of which were higher in the epilepsy group.

In the 39 cases $(33.62 \%)$ of viral encephalitis with epilepsy, there were 25 case $(64.10 \%)$ in which the main form was generalized seizure, which is consistent with current domestic reports. ${ }^{[9]}$ There were 7 cases of status epilepticus, 2 cases of simple partial seizures, 5 cases of complex partial seizures, 7 cases of generalized seizures secondary to partial seizures. In the 32 cases of non-status epilepsy, there were 12 cases whose duration of seizure was within $1 \mathrm{~min}, 8$ with duration of 1 to $5 \mathrm{~min}, 8$ with duration of 5 to $10 \mathrm{~min}, 1$ case with duration of 10 to $20 \mathrm{~min}$, and another 3 cases with unknown duration. There was a range in the number of epileptic attacks: 13 cases were once; 7 cases were 1 to 5 times; 4 cases were 5 to 10 times; and 8 cases were more than 10 times.

\section{Examination}

\section{CSF examinations}

All patients received a lumbar puncture. There were 53 cases of patients $(45.69 \%)$ whose intracranial pressure was higher than $180 \mathrm{mmH}_{2} \mathrm{O}$, including 9 cases of patients whose intracranial pressure was more than $300 \mathrm{mmH}_{2} \mathrm{O}$. The mean encephalic pressure was $184.10 \pm 64.29 \mathrm{mmH}_{2} \mathrm{O}$ in the group with epilepsy and $192.92 \pm 70.65 \mathrm{mmH}_{2} \mathrm{O}$ in the group without epilepsy. There are non-significant discrepancies between the two groups in encephalic pressure but the incidence of intracranial hypertension is lower than the study of Gong et al., ${ }^{[10]}$ which is $58.7 \%$. On the one hand, some patients received a lumbar puncture in the early stages of disease, and the intracranial pressure did not increase remarkably; on the other hand, some patients had been using drugs to reduce intracranial pressure before the lumbar puncture.

No clot was found in CSF routine examination. Only 1 case of patients with epilepsy manifests with yellow color and slightly mixed, 4 cases of patients were pale red, 111 cases of patients were colorless and transparent. There was only one case whose protein content in CSF was less than $0.15 \mathrm{~g} / \mathrm{L}$, and 38 cases of patients whose protein content was between 0.15 and $0.45 \mathrm{~g} / \mathrm{L}$, and 77 cases of patients whose protein content was above $0.45 \mathrm{~g} / \mathrm{L}$. There were 12 cases whose glucose content in CSF was less than $2.5 \mathrm{mmol} / \mathrm{L}$, and 93 cases of patients whose glucose content was between 2.5 and $4.5 \mathrm{mmol} / \mathrm{L}$, and 11 cases whose glucose content was above $4.5 \mathrm{mmol} / \mathrm{L}$. There were 30 cases of patients whose chloride in CSF was less than $120 \mathrm{mmol} / \mathrm{L}$, and 80 cases of patients whose chloride was between 120 and $132 \mathrm{mmol} / \mathrm{L}$. There were non-significant differences between the two groups in protein and chloride. However, there was significant difference between the two groups in glucose $(P=0.001)$, with higher glucose in those with epilepsy. Cytological examination of the CSF showed 95 cases with mainly increased lymphocytes, 16 cases were normal, 4 cases were mainly neutrophil predominant, and 1 case with elevated mononuclear cells. One percent of the phagocytes were found in one case and the same was true for granulocytes and plasma cells. Four cases were neutrophil predominant, 3 of them were from the group with epilepsy and one from the group without epilepsy. A lumbar puncture was performed in the early stages of their disease, and in this stage part of patients were mainly neutrophil predominant. With the treatment and development of disease, lymphocytes and monocytes were gradually increased and prevailed. However, this phenomenon is rarely seen in clinical practice because patients visit the hospital out of this period [Table 3].

\section{Imaging examination}

Brain imaging of viral encephalitis patients, using CT or MRI, showed foci mainly located in one or both temporal lobes, followed by frontal lobe, parietal lobe, occipital lobe, insula, hippocampus, and other parts of involvement [Table 4]. There were significant differences between the two groups in cortical involvements $(P=0.001)$ in which patients with epilepsy were higher [Table 5].

\section{EEG examination \\ According to the book of Clinical Electroencephalography written by Feng, ${ }^{[11]}$ EEG}

Table 3: Comparison of biochemical and cytological examination of cerebrospinal fluid between the two groups

\begin{tabular}{|c|c|c|c|c|}
\hline CSF & $\begin{array}{l}\text { With epilepsy group } \\
\qquad \mathrm{M}\left(\boldsymbol{P}_{25}, \boldsymbol{P}_{75}\right)\end{array}$ & $\begin{array}{l}\text { Without epilepsy group } \\
\qquad \mathrm{M}\left(\boldsymbol{P}_{25}, \boldsymbol{P}_{75}\right)\end{array}$ & $\mathbf{Z}$ & $\boldsymbol{P}$ \\
\hline CSF protein (g/L) & $0.53(0.34,0.87)$ & $0.58(0.43,0.85)$ & -0.885 & 0.376 \\
\hline CSF glucose (mmol/L) & $3.40(3.10,4.20)$ & $3.03(2.76,3.45)$ & -3.350 & 0.001 \\
\hline CSF chloride (mmol/L) & $122.60(116.00,125.70)$ & $123.00(121.15,125.00)$ & -0.790 & 0.430 \\
\hline Leukocyte $\left({ }^{*} 10^{6} / \mathrm{L}\right)$ & $61.00(25.00,150.00)$ & $37.00(11.00,92.50)$ & -2.237 & 0.025 \\
\hline Lymphocyte (\%) & $0.84(0.66,0.90)$ & $0.89(0.81,0.94)$ & -1.901 & 0.057 \\
\hline Monocyte (\%) & $0.09(0.06,0.14)$ & $0.08(0.05,0.15)$ & -0.763 & 0.445 \\
\hline Neutrophils (\%) & $0.04(0.01,0.18)$ & $0.02(0.00,0.05)$ & -1.620 & 0.105 \\
\hline
\end{tabular}

CSF: cerebrospinal fluid 
examination is divided into five grading standards, that is, normality, marginal state, mild abnormality, moderate abnormality, and severe abnormality. The abnormal rate of EEG is $37.07 \%$ (43 cases) in my research including 10 cases with epileptiform discharge. There were significant discrepancies between the two groups in abnormality of electroencephalogram $(P=0.001)$ in which patients with epilepsy are more severe [Table 6].

\section{Treatment}

All viral encephalitis patients were given antivirotic treatment. Ninety-nine cases were treated with acyclovir, a broad spectrum antiviral drug, which is a purine nucleoside analog that competitively inhibits the binding of 2'-deoxyuridine to block viral DNA synthesis and interfere with viral replication. The dose is generally $0.5 \mathrm{~g} \mathrm{Q} 8 \mathrm{~h}$ and treatment for 2 to 3 weeks, critically ill patients can be extended according to their condition. The main adverse reactions are acute renal failure, elevated transaminase, or encephalopathy in patients with normal renal function (seizures, dysarthria, hallucinations, etc.). ${ }^{[12]}$ Seventeen cases of patients were treated with ganciclovir, a derivative of acyclovir with the same mechanism of action. The conventional dose is $150 \mathrm{mg} \mathrm{Q12} \mathrm{h}$, and 10-14 days for a course of treatment. The main adverse reactions are for leukopenia and kidney damage.$^{\left[{ }^{[13]}\right.}$ It is reported that interferon can activate natural killer cells and macrophages, enhance B cell and $T$ cell activity, and slow viral replication. However, patients in my research have not used interferon due to its potential to aggravate nausea and vomiting, and, besides, it is expensive. Dehydration is induced in patients with intracranial hypertension to reduce intracranial pressure as well. Glucocorticoids have powerful anti-inflammatory and edema alleviating effects and are used to treat severe patients with white matter demyelination and severe brain edema. Glucocorticoid treatment is accompanied with potassium and calcium supplement and inhibition of gastric acid secretion to protect the digestive tract. Immunoglobulin can neutralize the virus, enhance immune cells, kill cells, neutralize antibodies, and other immune protection and anti-infective function. ${ }^{[14]}$ But immunoglobulin is expensive and has adverse reactions, such as, allergy, transmission of blood diseases, and kidney damage. Therefore, it is currently used for rapidly progressing and severe encephalitis. One case complicated with Guillain-Barre Syndrome developed quickly and was given immune globulin. If necessary, we give electrolyte replacement and rehydration, oxygen treatment, respiratory and circulation support, etc. Among the 39 cases of viral encephalitis complicated with epilepsy, and there were 11 cases $(28.20 \%)$ of patient using antiepileptic drugs (AEDs). There were 18 cases $(46.15 \%)$ using magnesium valproate to treat epilepsy, and 12 cases $(30.77 \%)$ using

Table 4: Comparison of imaging data between the two groups

\begin{tabular}{lcccc}
\hline \multirow{2}{*}{ Imaging examination } & \multicolumn{2}{c}{ With epilepsy group } & \multicolumn{2}{c}{ Without epilepsy group } \\
\cline { 2 - 5 } & Case & Rate (\%) & Case & Rate (\%) \\
\hline Frontal lobe & 7 & 16.67 & 8 & 14.55 \\
Parietal lobe & 5 & 11.90 & 3 & 5.45 \\
Occipital lobe & 4 & 9.52 & 3 & 5.45 \\
Temporal lobe & 16 & 38.10 & 19 & 34.55 \\
Insular & 3 & 7.14 & 3 & 3.45 \\
Brainstem & 0 & 2.38 & 2 & 0.20 \\
Basal ganglia, thalamus, lateral ventricles & 1 & 4.76 & 3 & 5.45 \\
Corpus callosum & 2 & 0 & 1 & 1.82 \\
Ventricle & 0 & 7.14 & 1 & 1.82 \\
Hippocampus & 3 & 2.38 & 1 & 1.82 \\
Cerebellum & 1 & & 3 & \\
\hline
\end{tabular}

Table 5: Comparison of cortical involvements between the two groups

\begin{tabular}{lcccccc}
\hline \multirow{2}{*}{ Imaging examination } & \multicolumn{2}{c}{ With epilepsy group } & \multicolumn{2}{c}{ Without epilepsy group } & \multirow{2}{*}{$\chi^{2}$} & \multirow{2}{*}{$\boldsymbol{P}$} \\
\cline { 2 - 5 } & Case & Rate (\%) & Case & Rate (\%) & \multirow{2}{*}{0.001} \\
\hline Cortical involvements & 23 & 58.97 & 20 & 25.97 & 12.085 & \multirow{2}{*}{0.001} \\
Cortical not involved & 16 & 41.03 & 57 & 74.03 & \\
\hline
\end{tabular}

Table 6: Comparison of EEG examination between the two groups

\begin{tabular}{|c|c|c|c|c|c|c|}
\hline \multirow{2}{*}{ EEG examination } & \multicolumn{2}{|c|}{ With epilepsy group } & \multicolumn{2}{|c|}{ Without epilepsy group } & \multirow{2}{*}{$\mathbf{Z}$} & \multirow{2}{*}{$\boldsymbol{P}$} \\
\hline & Case & Rate (\%) & Case & Rate (\%) & & \\
\hline The normality & 16 & 41.03 & 57 & 74.03 & & \\
\hline The marginal state & 1 & 2.56 & 3 & 3.90 & & \\
\hline Mild abnormality & 1 & 2.56 & 6 & 7.79 & -3.967 & 0.001 \\
\hline Moderate abnormality & 16 & 41.03 & 9 & 11.69 & & \\
\hline Severe abnormality & 5 & 12.82 & 2 & 2.59 & & \\
\hline
\end{tabular}

EEG: electroencephalogram 
Table 7: Comparison of prognosis between the two groups

\begin{tabular}{|c|c|c|c|c|c|c|}
\hline \multirow{2}{*}{ Prognosis } & \multicolumn{2}{|c|}{ With epilepsy group } & \multicolumn{2}{|c|}{ Without epilepsy group } & \multirow{2}{*}{$\mathbf{Z}$} & \multirow{2}{*}{$\boldsymbol{P}$} \\
\hline & Case & Rate (\%) & Case & Rate (\%) & & \\
\hline $\begin{array}{l}\text { Cure } \\
\text { Improved }\end{array}$ & $\begin{array}{c}0 \\
35\end{array}$ & $\begin{array}{c}0 \\
89.74\end{array}$ & $\begin{array}{l}15 \\
56\end{array}$ & $\begin{array}{l}19.48 \\
72.72\end{array}$ & & \\
\hline Aggravate & 1 & 2.56 & 1 & 1.30 & -2.443 & 0.015 \\
\hline Death & 0 & 0 & 1 & 1.30 & & \\
\hline Others & 3 & 7.69 & 4 & 5.19 & & \\
\hline
\end{tabular}

phenobarbital, 4 cases (10.26\%) using lamotrigine, 2 cases (5.13\%) using levetiracetam, 2 cases $(5.13 \%)$ using topiramate, and 1 case $(2.56 \%)$ using sodium valproate. There were 7 cases $(17.95 \%)$ using diazepam to prevent generalized seizure or status epilepticus. Most seizures can be terminated after treatment with one or two kinds of antiepileptic drugs; however, there were still 2 cases with status epilepticus terminated by midazolam or propofol.

\section{Prognosis}

According to the standard of prognosis, ${ }^{[15]}$ discharge situation are divided into five conditions, that is, cure, on the mend, aggravated, death, others (due to various reasons such as the interruption of treatment).

(1) Cure: all indexes including the symptoms, signs, related auxiliary examination results return to normal; (2) improved: at least one index including symptoms, signs, related auxiliary examination did not return to normal; (3) aggravated: exacerbations; (4) death; (5) others: treatment is interrupted due to the patient's own and other reasons. The discharge situation of the two groups were statistically analyzed with Rank Sum test and the results proved that there were significant discrepancies between the two groups $(P=0.015)$. The prognosis of patients with epilepsy was poorer [Table 7].

\section{DISCUSSION}

Viral encephalitis can occur in any season and at any age. Viral encephalitis frequently manifests with seizures. Its etiology and pathogenesis is not yet entirely clear. The pathogenesis has been found as follows: (1) in acute phase, many pathological changes can be found in viral encephalitis including the release of toxins and viral metabolites accumulation, cerebral edema, cortical arteriovenous thrombosis. All of these pathological changes can reduce the stability of cell membrane and cause epileptic seizure. Electrolyte disorders and excessively dehydration may also increase the incidence of epilepsy; (2) in the later stage, epileptogenic focus is formed in the brain. The manifestations of epileptogenic focus are neuron loss and disorganization because of cerebrocellular necrosis and gliocyte hyperplasia. Metabolic disorders of neurons, owing to ischemia, result in functional disorder ofion pumps across the membrane, resulting in the outflow of potassium ions, inflow of calcium, and sustained depolarization, which can cause seizures; ${ }^{[16]}$ (3) when the hypothalamus is involved, the abnormal secretion of antidiuretic hormone leads to fluctuations of blood sodium, which can further cause brain dysfunction and cause epileptic seizures. ${ }^{[17]}$

There were non-significant differences between the two groups in encephalic pressure, chloride, protein, and cytologic examination, but the patients with epilepsy had higher levels of glucose. At present, there is no relevant research on this feature. The view that hyperglycemia leads to epileptic seizures has been generally accepted. However, whether glucose in the CSF of epileptic patients is increased or not and what the mechanism might be still needs further research. Brain imaging examination showed that cortical involvements in patients with epilepsy were higher. Therefore, we should be alert to seizures in patients with cortical lesion. EEG examination shows that patients with epilepsy are more severe. We also should be alert to seizures in patients with moderate and severe abnormal EEG. Just as these researches about prognosis of viral encephalitis report, the severer the EEG manifestation, the worse the prognosis. ${ }^{[18]}$ EEG examination plays an important role in the treatment and prognosis of viral encephalitis because EEG changes are usually consistent with changes in patients' conditions. Non-convulsive seizures and nonconvulsive status epilepticus can aggravate the severity of dementia in patients with severe encephalitis. Nonconvulsive seizures are detectable only by continuous electroencephalographic monitoring, so EEG is particularly important. ${ }^{[19]}$ Conscious disturbance is a common manifestation of brain damage in encephalitis patients during the acute stage. We find that the proportion of patients with conscious disturbance and cognitive impairment in the epilepsy group was higher than that in the non-epileptic group. Therefore, an episode of viral encephalitis complicated with seizures aggravates conscious disturbance and cognitive impairment. Bartolomei et al. ${ }^{[20]}$ reviewed the research about the relationship between conscious disturbance and seizures over the past 50 years, and found that patients with seizures complicated with conscious disturbance have poor prognosis. The prognosis of patients with epilepsy is poorer if these izures are more frequent and/or longer duration, or the conscious 
disturbance in the acute phase is severer than for other patients. ${ }^{[21]}$ Therefore, this study suggests taking active antiepileptic treatment for patients with frequent seizures. As we lack follow-up investigation of viral encephalitis patients after discharge, we are unable to determine which AEDs have definite advantage over the others. The subject of viral encephalitis complicated with epilepsy remains for further study.

\section{Authors' contributions}

Concept: S.Y. Chen, W. Ma, Y.Y. Chen, X.W. Fan

Design: S.Y. Chen, X.W. Fan

Literature search: S.Y. Chen, W. Ma, Y.Y. Chen

Data analysis: S.Y. Chen

Manuscript preparation: S.Y. Chen, X.W. Fan

Manuscript editing: S.Y. Chen, X.W. Fan

Manuscript review: S.Y. Chen, X.W. Fan

\section{Financial support and sponsorship} None.

\section{Conflicts of interest}

There are no conflicts of interest.

\section{Patient consent}

Patient consent was obtained for publishing this work.

\section{Ethics approval}

Ethics committee approval was obtained for publishing this work.

\section{REFERENCES}

1. Hinson VK, Tyor WR. Update on viral encephalitis. Curr Opin Neurol 2001;14:369-74.

2. Zhang J, Zhang HL. Advances in the etiology and epidemiology of viral encephalitis. Chin J Viral Dis 2011;6:471-6.

3. Yan B, Zhang JT, Zhao W. Viral encephalitis: an analysis of cerebrospinal fluid from 124 cases. Acad J Chin PLA Med Sch 2014;5:430-2.
4. Shen YQ. Application value analysis of EEG in the diagnosis of viral encephalitis. Chin Mod Med 2015;5:96-7, 100.

5. Peng J, Luo TY, Lu FJ, Ouyang Y, Fang WD, Wu JQ. MRI, DWI and 1 H-MRS features of viral encephalitis: report of 36 cases. Acta Academiae Medicinae Militaris Tertiae 2009;3:257-60.

6. Qiao XH, Tong MQ, Yang F, Wang J, Ding CY. Research status of viral encephalitis complicated with epilepsy. Chin J Clinicians (Electronic Edition) 2012;11:3025-8. (in Chinese)

7. Misra UK, Kalita J. Seizures in encephalitis: predictors and outcome. Seizure 2009;18:583-7.

8. Singh TD, Fugate JE, Hocker SE, Rabinstein AA. Postencephalitic epilepsy: clinical characteristics and predictors. Epilepsia 2015;56:133-8.

9. Huang D, Huang YL, Liu T. Clinical characteristics of viral encephalitis patients with secondary epilepsy. Chin Tropical Med 2014;4:486-7, 495.

10. Gong SH, Cao ZH, Song JH. On examination of cerebrospinal fluid of viral encephalitis patients. $J$ Brain Nerv Dis 2008;6:694-6.

11. Feng YK. Clinical Electroencephalography. Beijing: People's Medical Publishing House; 1980. p. 62-4.

12. Whitley RJ, Gnann JW. Viral encephalitis: familiar infections and emerging pathogens. Lancet 2002;359:507-13.

13. Kuramoto T, Daikoku T, Yoshida Y, Takemoto M, Oshima K, Eizuru Y, Kanda Y, Miyawaki T, Shiraki K. Novel anticytomegalovirus activity of immunosuppressant mizorihine and its synergism with ganciclovir. J Pharmacol Exp Ther 2010;333:816-21.

14. Dong YP. Clinical observation of methylpredinsolone combined with immunoglobulin in the treatment of severe viral encephalitis. Clin Med 2014;5:20-1.

15. Zhen HJ, Qiu YQ, Zhu PQ. Clinical analysis of 431 cases of central nervous system infection. Chin J Infectious Dis 2006;3:190-1.

16. Usha KM, Chong T, Jayantee K. Viral encephalitis and epilepsy. Epilepsia 2008;49:S13.

17. Shao DB,Sun HC. Clinical progress of viral encephalitis. Chin Gen Pract 2008;20:1817-9.

18. Ai TM. Analysis of prognostic factors in 67 patients with viral encephalitis. Mod J Integr Traditional Chin West Med 2014;35:3934-6.

19. Li T, ShiLN, Fan XW. Diagnosis and treatment value of video electroencephalogram on non-convulsive status epilepticus of severe viral encephalitis. Ningxia Med $J$ 2015;8:686-8.

20. Bartolomei F, McGonigal A, Naccache L. Alteration of consciousness in focal epilepsy: the global workspace alteration theory. Epilepsy Behav 2014;30:17-23.

21. Ma HY, Zhang YF, Di Q. Prognosis and its influencing factors of postencephalitic epilepsy. J Clin Neurol 2016;2:120-3. 